\title{
Simulation of translational piezoelectric microactuators with application in miniaturized robots
}

\section{Simulation of translational piezoelectric microactuators with application in miniaturized robots}

Marta Vílchez Monge'

Estuardo Herrera Muñoz ${ }^{2}$

Natalia Rodríguez Rodríguez ${ }^{3}$

Paola Vega Castillo ${ }^{4}$

Fecha de recepción: 20 de enero del 2012

Fecha de aprobación: 19 de marzo del 2012

Vílchez, M; Herrera, E; Rodríguez, N; Vega, P. Simulation of translational piezoelectric microactuators with application in miniaturized robots. Tecnología en Marcha. Vol. 25, No 5.

Pág |24- 30.

Física. Escuela de Física, Tecnológico de Costa Rica. Teléfono: 8992-7134. Correo electrónico: mvilchez@itcr.ac.cr

2 Ingeniero en Electrónica. Escuela de Ingeniería Electrónica,Tecnológico de Costa Rica. Teléfono: 8827-5479 Correo electrónico: estuardohm@hotmail.com

3 Ingeniera en Electrónica. Escuela de Ingeniería Electrónica,Tecnológico de Costa Rica. Teléfono: 8995-3937. Correo electrónico: natisofl 8@hotmail.com

4 Doctora en Microelectrónica. Escuela de Ingeniería Electrónica, Tecnológico de Costa Rica. Teléfono: 2550-9248. Correo electrónico: pvega@itcrac.cr

Este artículo cuenta con el aval de la Vicerrectoría de Investigación y Extensión del Tecnológico de Costa Rica 


\section{Resumen}

En el diseño de robots miniaturizados deben evaluarse diferentes tipos de actuadores microelectromecánicos para determinar cuál es el más apropiado para cada función específica así como obtener el mejor efecto de actuación para el tamaño, fuerza, tensión de alimentación, energía, precisión y grados de libertad requeridos. El efecto piezoeléctrico es uno de los efectos más promisorios para incorporar microactuadores en robots miniaturizados. Este artículo presenta una breve comparación de métodos de actuación para desplazamiento translacional y se discute la factibilidad de su aplicación en robots miniaturizados. Se describe el caso específico de la evaluación de un actuador piezoeléctrico translacional utilizando COMSOL Multiphysics, así como un ejemplo de aplicación de una micropinza para ser incorporada en un robot miniaturizado con el fin de que logre la sujeción de objetos submicrométricos.

\section{Palabras clave}

Sistemas microelectromecánicos, MEMS, microactuador, método de elementos finitos, simulación, robot miniaturizado, micropinza.

\begin{abstract}
In the design of miniaturized robots, different types of microelectromechanical actuators must be evaluated to determine the most appropriate one for each specific function as well as the best actuation effect for the size, force, supply voltage, energy, precision and degrees of freedom required. The piezoelectric effect is one of the most promising effects to incorporate microactuators in miniaturized robots. This paper presents a brief comparison on actuation methods for translational displacement and discusses its feasibility for application in miniaturized robots. The specific case of evaluation of a translational piezoelectric actuator using $\mathrm{COMSOL}$ Multiphysics is described, as well as an application example for a microgripper to be incorporated in a miniaturized robot for grasping submicrometric objects.
\end{abstract}

\section{Key words}

Microelectromechanical systems, MEMS, microactuator, Finite Element Method, simulation, miniaturized robot, microgripper. 


\section{Introduction}

Although many different kinds of microactuators exist, only some of them actually adapt to the requirements of miniaturized robots. In the design of miniaturized robots, different types of microelectromechanical actuators must be evaluated to determine the most appropriate one for each specific function. The best actuation effect must be determined considering the size, force, supply voltage, energy, precision, linearity and degrees of freedom required.

Possible effects to implement translational microactuators include electrostatic,magnetostrictive, thermomechanical, piezoelectric and shape memory alloys.

Electromagnetic microactuators rely on induction, and therefore those require fabrication of microinductors which limits the scalability of the microactuator. In addition to that, electromagnetic effects scale unfavorably as size is reduced.

Electrostatic-based actuators are very common in MEMS applications. Typical devices such as the combdrive microactuators are relatively large. In order to increase their displacement, higher electric fields are required, leading to an increased actuation voltage.

Thermomechanical actuators generate displacement due to the thermal expansion of a heated material. Those actuators operate at low voltage, but their power consumption is high. Moreover, environmental influences can cool the actuator, thus making necessary to increase the power consumption to achieve the required operating temperature.

Shape memory alloys (SMA) contract those actuators in response to temperature due to a phase change; rearrangement of the atoms from one crystalline structure to another exerts a large force. Thus, SMAs can be attached to another structure to cause a displacement. Since the phase change is abrupt, SMAs are used for on-off actuators rather than for precise positioning. Moreover, achieving the phase change temperature requires high power consumption.

Parameter such as humidity and pressure operating conditions impose restrictions on the materials and actuation effects. Electroactive polymers have attracted attention for implementation of actuators and artificial muscles due to their low actuation voltages and biocompatibility, none the less, humid environments are required to operate; therefore pperation in vacuum conditions is not possible. Pneumatic microactuators based on gas or liquid flows may neither operate under vacuum conditions due to the high pressure difference between the vacuum environment and the actuator's microchambers.

Besides, the piezoelectric effect is one of the most promising effects to take into considerations when incorporating microactuators in miniaturized robots is considered. Piezoelectric actuators not only operate at low power consumption as well as relatively low supply voltages, thus can be directly driven by microcontrollers, but this actuators highly linear too. From the fabrication point of view, scalability is not a problem, and the actuators are relatively simple. Table I summarizes a brief comparison of actuation effects.

\section{Microactuator requirements}

In order to integrate the microactuator in the miniaturized robot, some requirements must be fulfilled:

- Maximum dimensions: $500 \mu \mathrm{m} \times 500 \mu \mathrm{m} \times 500 \mu \mathrm{m}$

- Minimum displacement: 300nm

- Power consumption: lower than ImW

- Supply voltage: $3 \mathrm{~V}$

- Operation in dry, vacuum environments 
Table I. Qualitative Comparison of Actuation Effects

\begin{tabular}{|c|c|c|c|c|c|c|}
\hline Actuation principle & $\begin{array}{c}\text { Supply } \\
\text { voltage }\end{array}$ & Size & Scalability & Linearity & $\begin{array}{c}\text { Power } \\
\text { consumption }\end{array}$ & Complexity \\
\hline Inductive & High & Large & Poor & Low & High & High \\
\hline Electrostatic & High & Large & Medium & Medium & Low & High \\
\hline Thermomechanical & Low & $\begin{array}{c}\text { Medium to } \\
\text { large }\end{array}$ & $\begin{array}{c}\text { Generally } \\
\text { poor }\end{array}$ & Low & High & Medium \\
\hline Piezoelectric & Low & Small & High & High & Low & Medium \\
\hline Shape memory alloy & Low & Large & Low & Low & High & Low \\
\hline
\end{tabular}

\section{Geometry description}

The geometry of the actuator is presented in figure I. It consists on a piezoelectric stack of 165 piezoelectric discs that have electrodes contacting each disc. The discs are $1 \mu \mathrm{m}$ thick and have $5 \mu \mathrm{m}$ radius. A piezoelectric stack actuator relies on the $d_{33}$ coupling mode and behaves linearly.

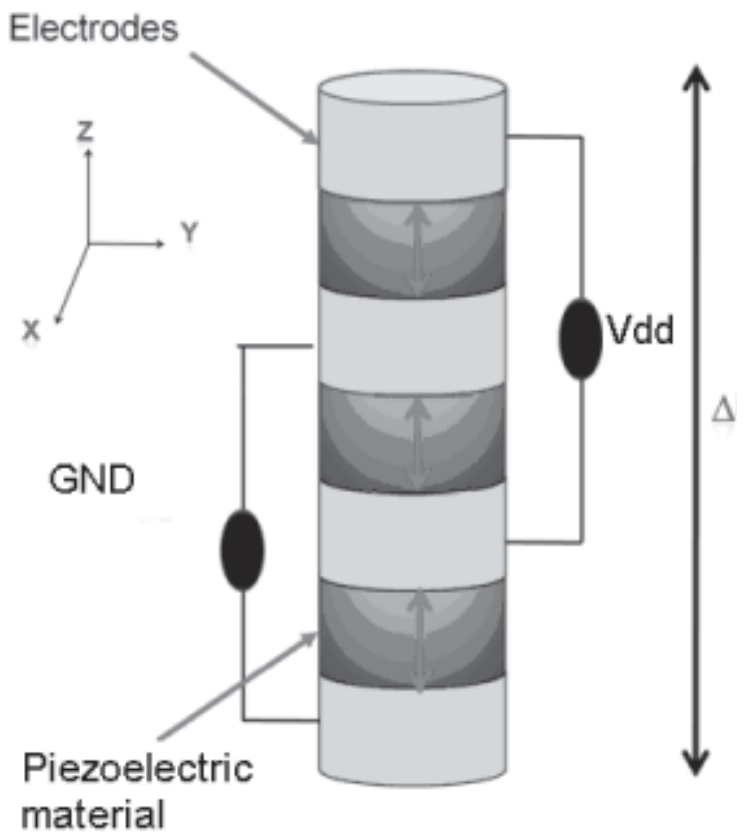

Figure I. Geometry of the microactuator.

\section{Microactuator modeling}

This section presents a description of the mathematical modelling of the microactuator and the chosen materials to achieve its specifications.

Lead zirconate titanate PZT-3203HD, with a $d_{33}$ constant, of $650 \mathrm{~m} / \mathrm{V}[1,2]$ is used on each piezoelectric. The piezoelectric charge constant $d$ is the polarization obtained per unit stress. It is also defined as the strain per unit of electric field and therefore is sometimes called strain coefficient. The subscript 33 indicates that both the deformation and the applied electric field have $z$-direction, as shown in figure 2.

The operating principle for the actuator is the inverse piezoelectric effect present in piezoelectric ceramics. This effect consists on a change of dimensions in the actuator due to an applied electric field. Actuator deformation, that is, elongation or contraction, depends on the direction of the applied electric field. In stack actuators, the cumulative effect of each disc leads to a total change in length which can be expressed as [3]

$\Delta L=d_{33} \cdot n \cdot V$

Where $\Delta L$ represents the total displacement experienced by the actuator, $d_{33}$ represents the material's longitudinal piezoelectric coupling coefficient, $n$ is the number of piezoelectric discs and $V$ is the applied voltage. The piezoelectric charge constants of PZT-3203HD are [I]

$d_{P Z T 3203 \mathrm{HD}}=\left(\begin{array}{cccccc}0 & 0 & 0 & 0 & 1000 & 0 \\ 0 & 0 & 0 & 1000 & 0 & 0 \\ -320 & -320 & 650 & 0 & 0 & 0\end{array}\right) 10^{-12} \mathrm{~m} / V$

\section{Microactuator simulation}

Some results for variables such as displacement and energy are presented here, as well as considerations and requirements for simulation.

Due to the stack configuration of the actuator, the polarization direction of each individual disc must be considered in the simulation. The material's polarization must be oriented in the same direction as the electric field to achieve elongation in the $z$ axis direction, as shown in figure 2. This leads to contraction in the xy plane. 


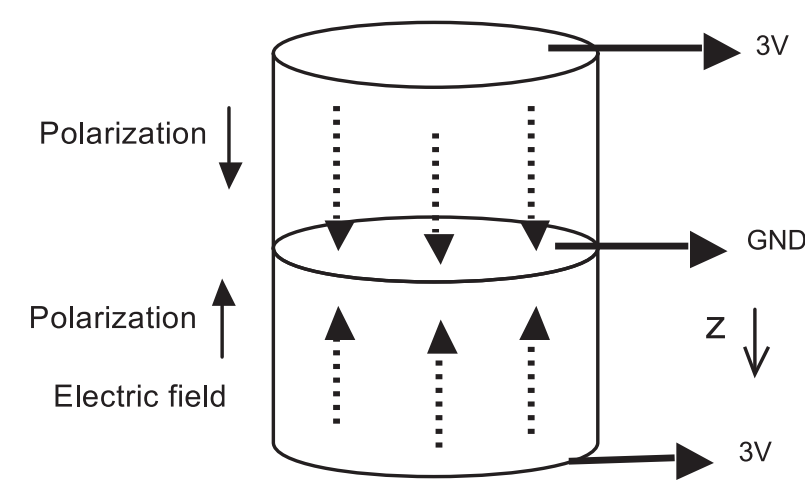

Figure 2. Direction of polarization and electric field required to obtain an elongation in the z-axis.

In order to obtain the best results in this effect, reorienting the material's polarization of all discs in the same direction of the electric field is fundamental, which can be done by configuring the Coordinate System option. By doing so, a new coordinate system with the $z$-axis rotated by $180^{\circ}$ is created. In addition to that, the boundary conditions were $3 \mathrm{~V}$ applied to the upper electrode of the stack, with OV and $3 \mathrm{~V}$ alternated on the rest of the electrodes. At the outer surface of each disc, a zero charge/simmetry condition is set, since no electric field is applied in that direction. Furthermore, the upper electrode of the stack is configured as a zero movement surface, since this plane is the fixing plane of the actuator to the robot.

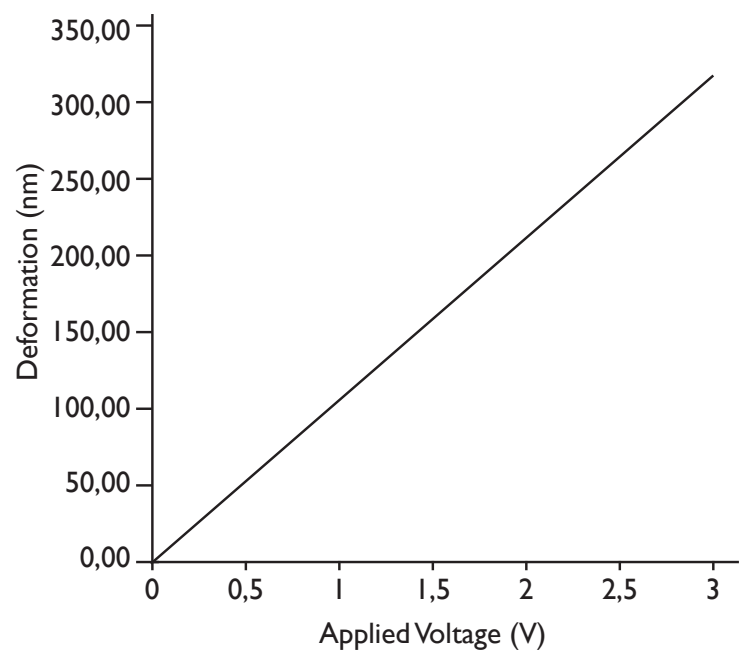

Figure 3. Microactuator deformation as a function of the applied voltage.
The actuator's displacement as a function of the applied voltage is presented in figure 3 , with a maximum elongation of $322 \mathrm{~nm}$ at $3 \mathrm{~V}$. The actuator elongates linearly at a rate of $10.74 \mathrm{~nm}$ per $100 \mathrm{mV}$. The actuator's energy density as a function of the applied voltage is presented in figure 4. Table I summarizes the simulation results obtained for this actuator.

Table 2. Summary of Simulation Results

\begin{tabular}{|l|l|}
\hline Parameter & Magnitude \\
\hline Maximum elongation $(\mathrm{nm})$ & 322 \\
\hline Force $(\mathrm{mN})$ & 11.54 \\
\hline Surface traction $\left(\mathrm{nN} / \mathrm{m}^{2}\right)$ & 14.7 \\
\hline Weight $(\mu \mathrm{N})$ & 3.91 \\
\hline Volume $\left(\mathrm{pm}^{3}\right)$ & 0.012 \\
\hline Maximum operating voltage $(\mathrm{V})$ & 3 \\
\hline Mass $(\mathrm{ng})$ & 399.42 \\
\hline Maximum energy consumption $(\mathrm{pJ})$ & 0.53 \\
\hline Maximum energy density $\left(/ / \mathrm{m}^{3}\right)$ & 151406 \\
\hline
\end{tabular}

\section{Application example: microgripper for grasping submicrometric objects}

One of the advantages of piezoelectric stacks is its ability to withstand high pressures. Stack actuators present the largest stiffness among piezoelectric actuators.

One possible application of the microactuator is the implementation of a microgripper for a miniaturized

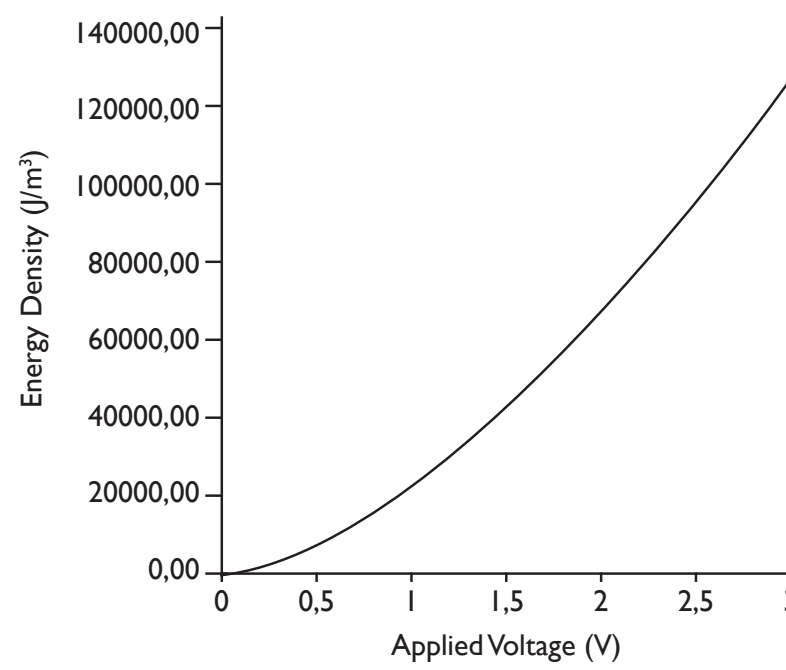

Figure 4. Microactuator energy density as a function of the applied voltage. 
robot. The gripper consists in two supporting Kapton bars, fixed to the robot's body. A piezoelectric stack of PZT-3203HD is attached to one of them. The gripper is normally closed. The piezoelectric stack contracts in response to the applied voltage, thus opening the gripper and allowing grasping objects. In order to hold the object grasped by the gripper, the voltage is set to $\mathrm{OV}$ and the microactuator elongates trying to return to the rest position. This procedure keeps the object pressed between the bar and the actuator as illustrated in figure 5. The geometry of the gripper is summarized in table 3 .

The $250 \mu \mathrm{m}$ center-to-center separation of the microactuator's bars corresponds to the pin pitch of a typical small size 805 I microcontroller, which would serve as attaching points for the microactuator and provide the voltage signal to drive the gripper. This ensures a short connection path to the electrodes of the piezoelectric stack.

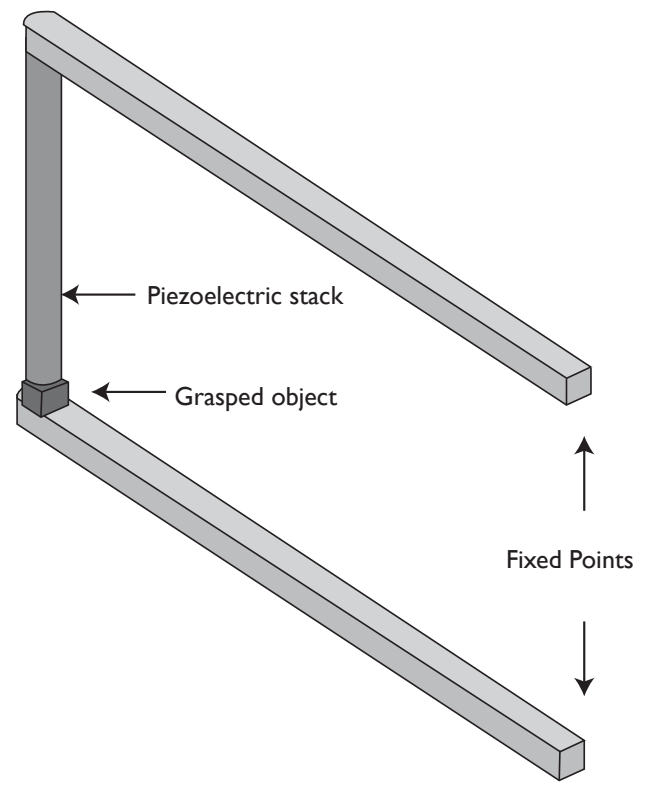

Figure 5. Piezoelectric stack-based gripper.

Table 3. Geometry of the Microgripper

\begin{tabular}{|l|l|}
\hline Characteristic| & Value \\
\hline Bar's Length $(\mu \mathrm{m})$ & 500 \\
\hline Bar's Pitch $(\mu \mathrm{m})$ & 250 \\
\hline Bar's height $(\mu \mathrm{m})$ & 15 \\
\hline Bar's thickness $(\mu \mathrm{m})$ & 20 \\
\hline Disc radius $(\mu \mathrm{m})$ & 10 \\
\hline Disc thickness $(\mu \mathrm{m})$ & $\mathrm{I}$ \\
\hline Number of discs & 235 \\
\hline
\end{tabular}

For this simulated geometry, the actuator reached piezoelectric stack displacement of $455 \mathrm{~nm}$ at $3 \mathrm{~V}$. Applied force and grasping were simulated for a cylindrical object with a radius of $5 \mu \mathrm{m}$ and $0.45 \mu \mathrm{m}$ thickness. The microgripper would be able to grasp objects with thicknesses between 50nm and 455nm. The actuator presented a power consumption of $1,39 \mathrm{~mW}$ operating at $10 \mathrm{kHz}$, with of $2.455 \mathrm{nF}$ capacitance.

The structural mechanics modules piezo solid and solid stress-strain of Comsol Multiphysics were used for the simulation. In order to simulate the force applied by the actuator to grasped objects, contact pairs must be defined to indicate the simulator that the objects will be in mechanical contact. For a $455 \mathrm{~nm}$-thick object, the compression simulated force on the grasped object yields $95 \mathrm{nN}$.

\section{Conclusions}

Piezoelectric stack actuators are suitable for translational actuation where precision and linearity are important. These actuators are highly scalable, show low power consumption, can operate at relatively low supply voltages and therefore can be directly driven by microcontrollers. All these characteristics make these actuators suitable to become part of a miniaturized robot. The actuator was simulated using finite element method simulation using Comsol Multiphysics, which served as a tool to obtain the actuator's displacement, energy density, power consumption and force.A possible application of a piezoelectric stack microactuator for a simple microgripper was simulated.

\section{References}

[1] CTS Electronic constants. Piezoelectric constants for 3203HD. Available at www.ctscorp.com/components/ pzt/downloads/PiezoConstantMatrix.pdf.

[2] Stewart S. Mukherjee Binu K. Characterization of Piezoelectric Materials for Transducers. Jet Propulsion Laboratory, California Institute of Technology, Pasadena, California. Available at http://arxiv.org/ftp/ arxiv/papers/07 I I/07 I I.2657.pdf

[3]Piefort, V. Finite Element Modelling of Piezoelectric Active Structures. PhD dissertation (2000-200 I). Universiteé Libre de Bruxelles. Belgium. 


\section{Acknowledgements}

This project has been funded by Intel Costa Rica, the Ministry of Science and Technology (project number Fl-029-20 I0) and the Vicepresidency of Research of Instituto Tecnológico de Costa Rica (project number 5402-|360-240 |). 